TUM-T39-96-30

\title{
Polarized deuteron structure functions at small $x^{*}$
}

\author{
J. Edelmann, G. Piller and W. Weise \\ Physik Department, Institut für Theoretische Physik, Technische Universität München, D-85747 \\ Garching, Germany
}

\begin{abstract}
We investigate shadowing corrections to the polarized deuteron structure functions $g_{1}^{d}$ and $b_{1}$. In the kinematic domain of current fixed target experiments we observe that shadowing effects in $g_{1}^{d}$ are approximately twice as large as for the unpolarized structure function $F_{2}^{d}$. Furthermore, we find that $b_{1}$ is surprisingly large at $x<0.1$ and receives dominant contributions from coherent double scattering.
\end{abstract}

To be published in Z. Phys. A.

*Work supported in part by BMBF 
In recent unpolarized lepton-nucleus scattering experiments at CERN (NMC) and FNAL (E665) [1] nuclear shadowing at small values of the Bjorken scaling variable $x<0.1$ has been established as a leading twist effect. It is driven by the diffractive excitation of the (virtual) photon into hadronic states which interact coherently with several nucleons in the target nucleus.

Considering the growing interest in spin-dependent structure functions, a study of shadowing effects in polarized deep-inelastic scattering is urgently needed. In particular, the extraction of the neutron spin structure function $g_{1}^{n}$ from deuteron and ${ }^{3} \mathrm{He}$ data requires a detailed knowledge of nuclear effects in the small $x<0.1$ domain. Furthermore, planned experimental investigations of the yet unmeasured deuteron structure function $b_{1}$ [2] call for an analysis of its small $x$ behavior which is driven, as we will show, by coherent double scattering contributions. This short note summarizes our first results. A more complete and extended exposition of the material and the formalism is in preparation [3].

In the following we focus on the deuteron structure functions $F_{1,2}^{d}, g_{1}^{d}$ and $b_{1}$ at small values of the Bjorken scaling variable $x<0.1$. Based on the optical theorem which connects forward virtual Compton scattering and deep-inelastic scattering, these structure functions can be expressed in terms of (virtual) photon-deuteron helicity amplitudes $\mathcal{A}_{+h}^{d}$, where "+" denotes the helicity of the transversely polarized photon and $h=0,+,-$ labels the deuteron helicity «:

$$
\begin{aligned}
F_{1}^{d} & \sim \frac{1}{3} \operatorname{Im}\left(\mathcal{A}_{+-}^{d}+\mathcal{A}_{++}^{d}+\mathcal{A}_{+0}^{d}\right), \\
g_{1}^{d} & \sim \frac{1}{2} \operatorname{Im}\left(\mathcal{A}_{+-}^{d}-\mathcal{A}_{++}^{d}\right), \\
b_{1} & \sim \frac{1}{2} \operatorname{Im}\left(2 \mathcal{A}_{+0}^{d}-\mathcal{A}_{++}^{d}-\mathcal{A}_{+-}^{d}\right) .
\end{aligned}
$$

The deuteron helicity amplitudes can be split into contributions from the incoherent scattering off either the proton or neutron, and a term which accounts for the coherent scattering from both nucleons:

$$
\mathcal{A}_{+h}^{d}=\mathcal{A}_{+h}^{p}+\mathcal{A}_{+h}^{n}+\delta \mathcal{A}_{+h} .
$$


Since nuclear effects from binding and Fermi-motion are relevant only at moderate and large $x \gtrsim 0.2$ (e.g. see references in [5]) we neglect them in the following. Then the single scattering amplitudes $\mathcal{A}_{+h}^{p}$ and $\mathcal{A}_{+h}^{n}$ are directly related to the free proton and neutron structure functions, respectively. Note that in this approximation single scattering yields no contribution to $b_{1}$. The double scattering amplitude $\delta \mathcal{A}_{+h}$ is responsible for shadowing corrections in $F_{1,2}^{d}$ and $g_{1}^{d}$ and dominates $b_{1}$ at small $x$. Treating the deuteron target as a non-relativistic bound state, described by the helicity dependent wave function $\psi_{h}(\boldsymbol{r})$ we obtain:

$$
\begin{aligned}
\delta \mathcal{A}_{+h} & \sim \sum_{X} \int d^{3} r \int \frac{d^{3} k}{(2 \pi)^{3}} \psi_{h}^{\dagger}(\boldsymbol{r}) \mathcal{T}\left(\gamma^{*} p \rightarrow X p\right) \\
& \times \frac{e^{-i \boldsymbol{k} \cdot \boldsymbol{r}}}{\nu^{2}-\boldsymbol{k}_{\perp}^{2}-\left(q_{z}-k_{z}\right)^{2}-M_{X}^{2}+i \epsilon} \mathcal{T}\left(X n \rightarrow \gamma^{*} n\right) \psi_{h}(\boldsymbol{r})+[p \leftrightarrow n],
\end{aligned}
$$

where $q^{\mu}=\left(\nu, \mathbf{0}_{\perp}, q_{z}\right)$ is the four-momentum of the virtual photon. The sum is taken over all diffractively excited hadronic intermediate states with momentum $q-k$ and invariant mass $M_{X}$. The amplitudes

$$
\begin{gathered}
\mathcal{T}\left(\gamma^{*} p \rightarrow X p\right)=P_{\uparrow}^{p} t_{+\uparrow}^{\gamma^{*} p \rightarrow X p}+P_{\downarrow}^{p} t_{+\downarrow}^{\gamma^{*} p \rightarrow X p}, \\
\mathcal{T}\left(X n \rightarrow \gamma^{*} n\right)=P_{\uparrow}^{n} t_{+\uparrow}^{X n \rightarrow \gamma^{*} n}+P_{\downarrow}^{n} t_{+\downarrow}^{X n \rightarrow \gamma^{*} n}
\end{gathered}
$$

stand for proper combinations of projection operators $P_{\uparrow(\downarrow)}^{p(n)}$ onto proton (neutron) states with helicity $+1 / 2(-1 / 2)$, and $t_{+\uparrow}^{\gamma^{*} p \rightarrow X p}$ etc., the corresponding photon-nucleon helicity amplitudes for the diffractive production of the state $X$.

Coherent double scattering contributions to the deuteron structure functions are determined by the imaginary part of $\delta \mathcal{A}_{+h}$ which is dominated by the diffractive production and re-scattering of hadronic states in forward direction. The result for $\delta \mathcal{A}_{+h}$ can then be expressed in terms of helicity-dependent diffractive photon-nucleon forward amplitudes (which we assume to be purely imaginary), combined with the longitudinal deuteron form factor

$$
\mathcal{F}_{h}(1 / \lambda)=\int_{-\infty}^{\infty} d z\left|\psi_{h}\left(\mathbf{0}_{\perp}, z\right)\right|^{2} \cos (z / \lambda)
$$


for each deuteron helicity $h=0,+,-$. These form factors are functions of the inverse propagation length $\lambda^{-1}$; a hadronic fluctuation of mass $M_{X}$ can contribute to coherent double scattering only if its propagation length $\lambda=2 \nu /\left(Q^{2}+M_{X}^{2}\right)$ exceeds the deuteron size: $\lambda \gtrsim\left\langle r^{2}\right\rangle_{d}^{1 / 2} \approx 4 f m$.

\section{Unpolarized structure function}

Neglecting any spin- and isospin-dependence of the diffractive photon-nucleon amplitudes yields the standard result for shadowing in the unpolarized structure function $F_{1}^{d}=F_{1}^{p}+$ $F_{1}^{n}+\delta F_{1}($ e.g. see references in [5]):

$$
\delta F_{1}\left(x, Q^{2}\right)=-\left.\frac{Q^{2}}{x \pi \alpha} \int d M_{X}^{2} \frac{d^{2} \sigma^{\gamma^{*} N}}{d M_{X}^{2} d t}\right|_{t \approx 0} \mathcal{F}(1 / \lambda),
$$

where $\alpha=1 / 137$. Here $\mathcal{F}=\left(\mathcal{F}_{+}+\mathcal{F}_{-}+\mathcal{F}_{0}\right) / 3$ is the helicity averaged longitudinal deuteron form factor, and $d^{2} \sigma^{\gamma^{*} N} / d M_{X}^{2} d t$ is the unpolarized forward cross section for the diffractive

production of hadronic states $X$ from nucleons. Corrections to eq.(9) are discussed in [3] (see also [6]).

\section{Polarized structure function $\mathrm{g}_{1}^{\mathrm{d}}$}

In $g_{1}^{d}=\left(1-\frac{3}{2} \omega_{D}\right)\left(g_{1}^{p}+g_{1}^{n}\right)+\delta g_{1}$ the single scattering contribution is modified by the $D$-state probability $\omega_{D}$. Assuming isospin invariance of the unpolarized diffractive photonnucleon amplitudes leads to:

$$
\delta g_{1}\left(x, Q^{2}\right)=-\frac{Q^{2}}{4 x \pi \alpha} \int d M_{X}^{2}\left[\left.\frac{d^{2} \sigma_{+\downarrow}^{\gamma^{*} p}}{d M_{X}^{2} d t}\right|_{t \approx 0}-\left.\frac{d^{2} \sigma_{+\uparrow}^{\gamma^{*} p}}{d M_{X}^{2} d t}\right|_{t \approx 0}\right] \mathcal{F}_{+}(1 / \lambda)+[p \leftrightarrow n] .
$$

A direct comparison of the double scattering helicity amplitudes yields the following upper limit:

$$
\frac{\left|\delta g_{1}\right|}{F_{1}^{N}} \leq \frac{3\left|\delta F_{1}\right|}{2 F_{1}^{N}} \approx \frac{3\left|\delta F_{2}\right|}{2 F_{2}^{N}}
$$

where $F_{1,2}^{N}=\left(F_{1,2}^{p}+F_{1,2}^{n}\right) / 2$. Data on $\delta F_{2} / 2 F_{2}^{N}$ are available from E665 [1] (see Fig.1a). We conclude from eq.(11) that, for recent data analyses [7] on the neutron structure function $g_{1}^{n}$, uncertainties due to the shadowing correction $\delta g_{1}$ are within the experimental errors. Note that the upper bound (11) is not helpful at very small values $x<0.01$. 
In a laboratory frame description at $x<0.1$, the virtual photon fluctuates into a hadronic state which then interacts with one or several nucleons inside the nuclear target. In the kinematic range of currently available experimental data on shadowing in unpolarized lepton scattering, it has turned out to be a good approximation to consider the interaction of only one effective hadronic state with invariant mass $M_{X}^{2} \sim Q^{2}$ and a coherence length $\lambda \sim 1 / 2 M x$. This "one-state" approximation has been recently applied to shadowing in $g_{1}^{3} \mathrm{He}[8]$. For deuterium it yields:

$$
\frac{\delta g_{1}}{g_{1}^{N}}=\mathcal{R}_{g_{1}} \frac{\delta F_{2}}{F_{2}^{N}},
$$

with $\mathcal{R}_{g_{1}}=\mathcal{F}_{+}(2 M x) / \mathcal{F}(2 M x)$. At $x \lesssim 0.01$ we obtain for realistic deuteron wave functions $\mathcal{R}_{g_{1}}=2.7$ (Paris potential [10]) and $\mathcal{R}_{g_{1}}=2.4$ (Bonn one-boson exchange potential [11]). In Fig.1b we show the shadowing correction $\delta g_{1} / 2 g_{1}^{N}$ using recent data on $\delta F_{2} / 2 F_{2}^{N}$ from the E665 collaboration [ []] . It should be noted that for decreasing values of $x$ the experimental data for the shadowing ratio $\delta F_{2} / 2 F_{2}^{N}$ are taken at decreasing values of the average momentum transfer $\overline{Q^{2}}$. Therefore our result for $\delta g_{1}$ shown in Fig.1b corresponds, strictly speaking, to the fixed target kinematics of E665 [1] which is not far from the kinematics of SMC [7].

In the kinematic domain of recent experiments one has $\left|g_{1}^{N}\right|=\left|g_{1}^{n}+g_{1}^{p}\right| / 2<0.5$ [7] . Returning to eq.(12) one then observes that shadowing amounts to less then $5 \%$ of the experimental error on $g_{1}^{n}$ for the SMC analysis [1].

\section{Polarized structure function $b_{1}$}

At small values of $x<0.1$ coherent double scattering dominates $b_{1}$ and leads to:

$$
b_{1}\left(x, Q^{2}\right)=\left.\frac{Q^{2}}{x \pi \alpha} \int d M_{X}^{2} \frac{d^{2} \sigma^{\gamma^{*} N}}{d M_{X}^{2} d t}\right|_{t \approx 0}\left(\mathcal{F}_{+}(1 / \lambda)-\mathcal{F}_{0}(1 / \lambda)\right),
$$

where binding effects are small [4]. Here we have again neglected any spin- and isospindependence of the diffractive photon-nucleon amplitudes [3]. At small $x \lesssim 0.01$ a good ap-

proximation to eqs. (9. 10,13) is obtained by setting $\mathcal{F}_{h}(1 / \lambda) \approx \mathcal{F}_{h}(0)$ for hadronic states 
with $\lambda>\left\langle r^{2}\right\rangle_{d}^{1 / 2}$, while $\mathcal{F}_{h}(1 / \lambda)=0$ otherwise 9 . We then find for the double scattering contribution to $b_{1}$ :

$$
\frac{b_{1}}{F_{1}^{N}}=\mathcal{R}_{b_{1}} \frac{\delta F_{2}}{F_{2}^{N}}
$$

with $\mathcal{R}_{b_{1}}=\left(\mathcal{F}_{0}(0)-\mathcal{F}_{+}(0)\right) / \mathcal{F}(0)$. Note that $\mathcal{R}_{b_{1}}=0$ if the $D$-state admixture in the deuteron is neglected. We obtain $\mathcal{R}_{b_{1}}=-1.03$ (Paris potential [10]) and $\mathcal{R}_{b_{1}}=-0.58$ (Bonn one-boson exchange potential [1]). With recent data for $F_{2}^{N}$ [12 combined with the Callan-Gross relation, and the measured $\delta F_{2} / 2 F_{2}^{N}$ [1] we find a large contribution to $b_{1}$ from coherent double scattering as shown in Fig.2. This is a remarkable result. As we found after the present calculations [3] were finished, a similar conclusion has recently been reached in ref. [13].

In summary, we have presented first results for shadowing corrections in polarized deepinelastic scattering from deuterium. In the kinematic regime of current fixed target experiments, shadowing in $g_{1}^{d}$ is found to be approximately twice as large as for the unpolarized structure function $F_{2}^{d}$. Nevertheless it plays a minor role for the extraction of the neutron structure function $g_{1}^{n}$. Furthermore we find dominant contributions to the deuteron structure function $b_{1}$ at $x<0.1$ from coherent double scattering involving the deuteron $D$-state. 


\section{REFERENCES}

[1] NMC Collab., P. Amaudruz et al., Nucl. Phys. B441 (1995) 3; NMC Collab., A. Arneodo et al., Nucl. Phys. B441 (1995) 12; E665 Collab., M.R. Adams et al., Phys. Rev. Lett. 75 (1995) 1466; E665 Collab., M.R. Adams et al., Z. Phys. C67 (1995) 403

[2] H.E. Jackson, Prospects for HERMES Spin Structure Studies at HERA, in Proc. 11th Int. Symp. on High Energy Spin Physics, Bloomington, 1994, AIP Conf. Proc. 339

[3] J. Edelmann, Diploma thesis, Universität Regensburg, September 1996; J. Edelmann, G. Piller and W. Weise, in preparation

[4] P. Hoodbhoy, R.L. Jaffe and A. Manohar, Nucl. Phys. B312 (1989) 571; H. Khan and P. Hoodbhoy, Phys. Rev. C44 (1991) 1219

[5] M. Arneodo, Phys. Rep. 240 (1994) 301

[6] H. Khan and P. Hoodbhoy, Phys. Lett. B298 (1993) 181

[7] SMC Collab., D. Adams et al., Phys. Lett. B357 (1995) 248; E143 Collab., K. Abe et al., Phys. Rev. Lett. 75 (1995) 25

[8] L. Frankfurt, V. Guzey and M. Strikman, Phys. Lett. B381 (1996) 379

[9] G. Piller, G. Niesler and W. Weise, preprint TUM/T39-96-27, submitted to Z. Phys. A

[10] M. Lacombe, B. Loiseau, J.M. Richard and R.V. Mau, Phys. Rev. C21 (1980) 861

[11] R. Machleidt, K. Holinde and C. Elster, Phys. Rep. 149 (1987) 1

[12] E665 Collab., M.R. Adams et al., Phys. Rev. D54 (1996) 3006

[13] N.N. Nikolaev and W. Schäfer, preprint KFA-IKP-1996-19 


\section{FIGURES}

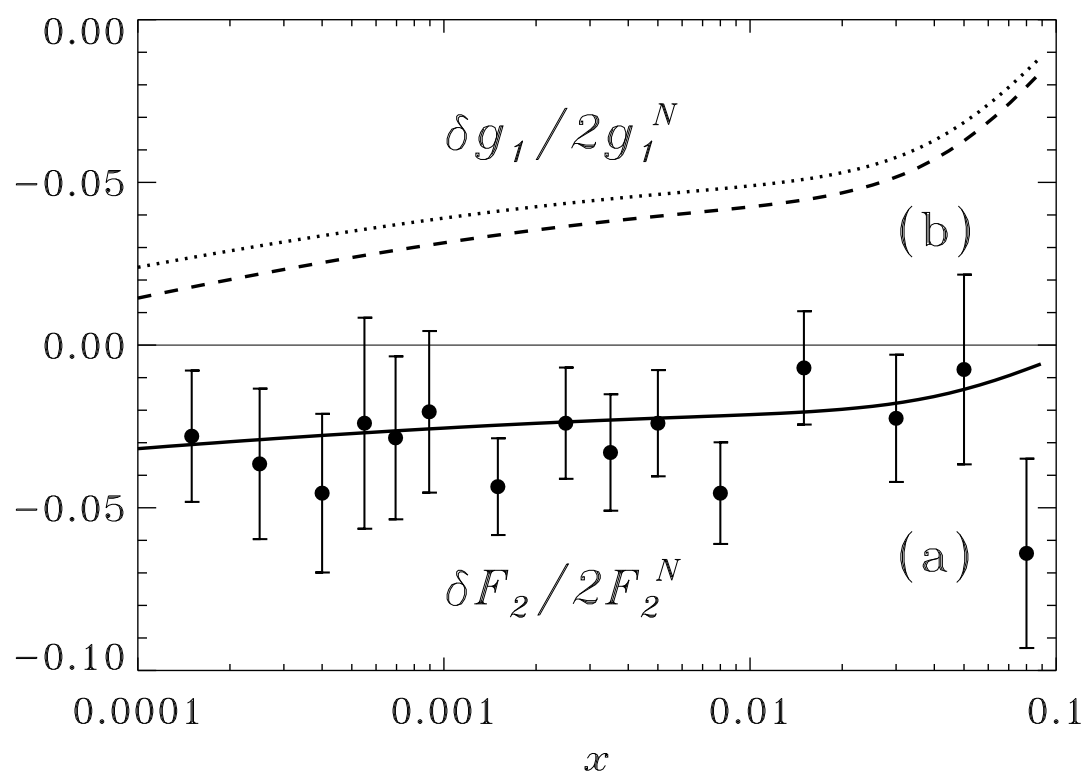

FIG. 1. (a) shadowing correction $\delta F_{2} / 2 F_{2}^{N}$, data from E665 [1]. The full line represents a parametrization of the data used in (12) and (14). (b) shadowing correction $\delta g_{1} / 2 g_{1}^{N}$ from (12). The dashed and dotted curves correspond to the Paris [10] and Bonn [11] potential respectively.

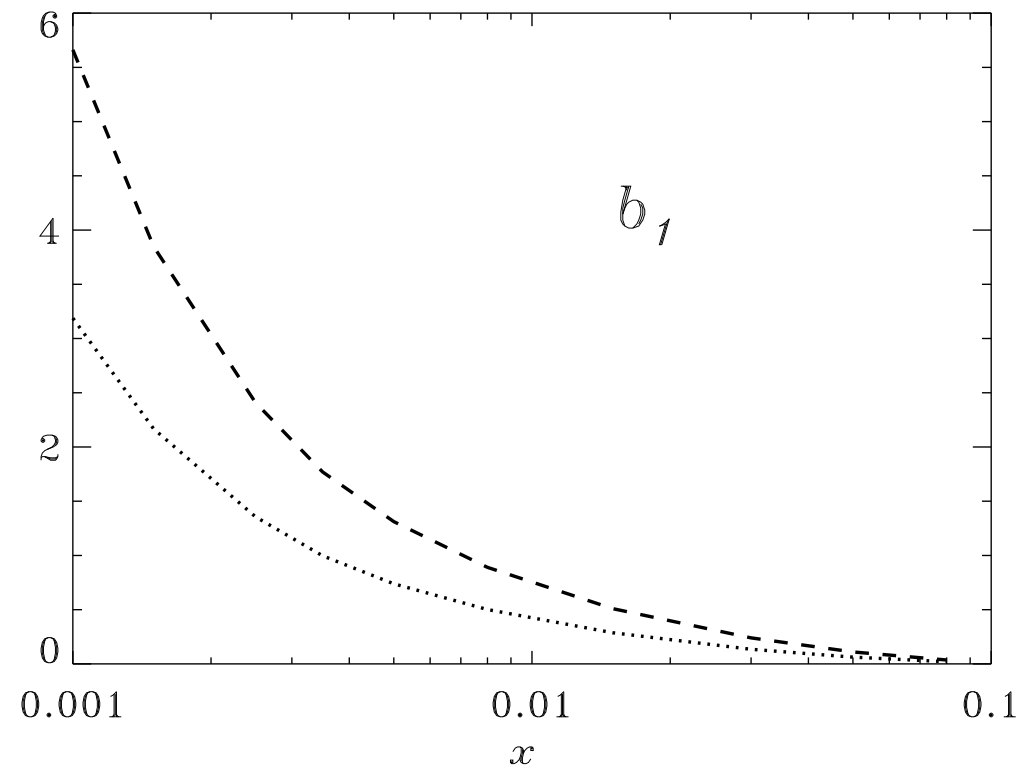

FIG. 2. Double scattering contribution to $b_{1}$ from (14). The dashed and dotted curves correspond to the Paris [10] and Bonn [11] potential respectively. 